\section{Roundtable on the Future of Nuclear Medicine Training}

TO THE EDITOR: In preparing this letter on the proposal of the American Board of Nuclear Medicine (ABNM)-American Board of Radiology (ABR) task force (1), my most difficult challenge was to reflect on my own career choices, the current state of my profession, and what perspective might add to the myriad prior review articles and committee statements regarding the field of nuclear medicine. I considered the Confucius quotes, "The beginning of wisdom is to call things by their proper name" and "Choose a job you love, and you will never have to work a day in your life," and decided that this letter should include the perspectives of several other individuals, as my enthusiasm for what I currently do should not cloud my choice of a "name" for its future direction. I asked three individuals-my chief resident (trainee), a lifetime diplomate and leader in the field, and a neutral health care management consultant-to join me. To complete this feat, the nuclear medicine physicians submitted their individual perspectives to the consultant, who formed a coherent summary and finally circled back to propose a brief set of action items agreed on by all four of us.

\section{J.R. OSBORNE}

\section{Personal Introduction}

I consider myself one of the first trainees in Steve Larson's "Janus project," the concept of combining related training perspectives (such as radiology) and scientific rigor into nuclear medicine (2). To that end, I am dual-certified by the ABNM and the ABR. I also completed a $\mathrm{PhD}$ degree in biochemistry at Columbia University before my postdoctoral training in molecular imaging at Memorial Sloan Kettering Cancer Center 13 years ago. Although the training period was long, I can see no better path to being the program director at Memorial Sloan Kettering Cancer Center and a clinician scientist. I am, however, conflicted about a concept that led to this letter: If this is my path, why has it worked for me whereas others are reluctant to go down the same road? If leadership is needed, how exactly do I lead others down a road that will result in career satisfaction? As Martin Luther King, Jr., said, "A genuine leader is not a searcher for consensus but a molder of consensus." It follows, then, that one should mold rather than search. And to paraphrase Winston Churchill, "The optimist sees the opportunity in every difficulty." So I am indeed in the right place.

\section{Perspective}

Motivation to enter training is both altruistic and financial. Trainees want to do what they enjoy but also need to know what may be at the end of the rainbow. The lack of jobs and role models has been and will continue to be a problem at the beginning of the pipeline. One can attract and influence the altruism of the aspiring medical student, but students should know what will be available to them in 10 years. I would suggest that if the influence peddlers in our profession are less than 10 years from retirement, their

COPYRIGHT (c) 2015 by the Society of Nuclear Medicine and Molecular Imaging, Inc. audience may be limited unless midcareer physicians who have intermediate timeline goals are in the conversation. A joint message affirmed by both categories of stakeholder is likely to be the optimal answer.

My sense is that our most recent focus on CT as the technology that separates the nuclear medicine resident from the radiology resident is misguided. The gap is considerably greater, and the maturation of MRI, ultrasound, fusion technologies, and big data makes this disconnect even more profound. We must shift the conversation from one of obvious deficits to one of obvious strengths (3), which must be actionable and reimbursable. The most clear and pressing need is investigational agents and therapy.

At the time of writing this letter, a joint ABR-ABNM task force proposal has been released for comment (June 3, 2015) and, if accepted, would create a singular pathway for the joint trainee. Admittedly, I had thought that such a proposal was not forthcoming and that the continued dual-board mission would remain for at least another few years. Although key details are missing from the proposal (which may be more apparent by the time this letter is in press), it would seem that the initial seeds have been planted by both boards.

If the path is then to be decided by the ABR and the nuclear medicine-diagnostic radiology trainee, perhaps there will be more uniformity in training. On the other hand, the past workforce diversity will be diminished by stakeholders entering from internal medicine and other paths. I suggest creating a biodesign-type impetus in which the trainees participate in the process of achieving the approval of the Food and Drug Administration and the Centers for Medicare and Medicaid Services for the most impactful imaging and therapy agents - this would change the field. The process would require an unprecedented effort of strategic planning, data sharing, and grassroots practical action. It would enhance the value of the appropriately credentialed stakeholder. Our current advantage is that we are relatively small. The construction of this type of effort would require a nucleus of like-minded physicians prepared to take a National Oncologic PET Registry approach to the next steps in the field. And unlike prior efforts, it would have to be bottom-up rather than top-down.

\section{BEYLERGIL}

\section{Personal Introduction}

I recently completed an Accreditation Council for Graduate Medical Education-accredited nuclear medicine residency preceded by a nuclear oncology fellowship at Memorial Sloan Kettering Cancer Center. Having had prior experience in nuclear medicine, I can safely state that I am a strong believer and advocate of nuclear medicine and molecular imaging. However, I decided to proceed with a diagnostic radiology residency. I had read Dr. Larson's excellent essay (2) on the Janus project and was well aware of the job market when I started my nuclear medicine residency. It is probably true that the job market for recent nuclear medicine graduates is dismal (4). Furthermore, only about a quarter of nuclear medicine studies performed in the United States are interpreted by nuclear physicians; the rest are shared by diagnostic radiologists and cardiologists (5), further decreasing job availability for nuclear physicians. However, my decision was not based solely on the job market or downward-trending figures; my ultimate motivation was to 
become a better hybrid imager over the long term. I believe that building one's own combined residency is a viable option for recent graduates of nuclear medicine programs, although not necessarily the most efficient one.

\section{Perspective}

Although there is a downward trend in the number of existing nuclear medicine residency programs, the emphasis on hybrid imaging and CT training has increased significantly in existing programs. However, cross training and certification for CT are only palliative measures that do not address future challenges. Although in its infancy, PET/MRI might change the current situation, requiring a major overhaul of existing cross-training. Joint programs leading to $\mathrm{ABR}$ and $\mathrm{ABNM}$ certifications seem to be a logical way to proceed over the long term, similar to the Arkansas experience (6).

Albert Einstein said, "In the middle of difficulty lies opportunity." Indeed, molecular imaging and therapy are exciting opportunities in the midst of existing challenges. Our department's name was recently changed to "Molecular Imaging and Therapy Service" to emphasize these opportunities and to acknowledge the changing scope of nuclear medicine. I believe there will always be academic jobs for nuclear physicians with academic goals and research interests. The practice of nuclear medicine in Europe is very different, and academic nuclear medicine is thriving thanks to advances such as peptide-based or prostate-specific membrane antigen-based theranostics.

However, private practice also has an important role in shaping our practice parameters and may prefer to hire radiologists simply because it is a more cost-effective decision when considering emergency and cross-modality coverage. Vendors also have a considerable influence on our practice. If a hybrid system is more cost-effective to manufacture, marketing efforts and further research will be channeled to that device; if a specific name for a device is better for marketing purposes, the device will be named accordingly.

Many concerns cited by trainees in Dr. Larson's 2011 essay are, unfortunately, still valid today. Both the SNMMI and the ABNM should work together to address these issues, and indeed, there has been significant effort and sincere attempts (7). In the meantime, I think that recent graduates of nuclear medicine residencies who do not want to proceed with radiology training should focus on an academic nuclear medicine career and exploit the advantages of molecular imaging and therapy. There will always be a place for well-qualified clinician scientists with adequate training in molecular imaging, theranostics, dosimetry, and pharmacokinetics. Academia and research have always been strong suits of nuclear medicine. To quote Dr. Larson, we may have to "give up the Escalade in the driveway," and that is okay-you can always hail one with your smart phone. If you are a trainee, it is also your responsibility to add unique qualifications to your resume and make it stand out among thousands. Medicine has never been a 9-to-5 job; rather, you sign up for lifelong learning when you choose medicine.

The good news is that the joint ABR-ABNM task force has started building a platform to resolve this issue. At the time of publication of this letter, this initiative might be closer to completion. The proposal involves eventual dissolution of the ABNM and creation of a new discipline of diagnostic radiology-nuclear medicine under the umbrella of the ABR. I think a consensus that will satisfy both parties is achievable. During this transition, appropriate actions should be taken to preserve vital clinical research in molecular imaging and targeted therapy.
Regardless of issues concerning reimbursement or the job market, fellow clinicians need us for critical decisions on patient care. A time may come when an omnipotent physician such as Dr. McCoy of the fictional series Star Trek will diagnose all ailments known to humankind using his handheld "tricorder." This would obviously result in a significant change in the practice of radiology. Even then, I would be confident in the ability of imaging professionals to adapt. To quote Albert Einstein again, "The measure of intelligence is the ability to change."

\section{M.M. GRAHAM \\ Personal Introduction}

I entered nuclear medicine after training in electrical engineering and biophysics. The biophysics training was done at the Donner Lab at the University of California, Berkeley, where Hal Anger was down the hall working on his next device. I received a letter of recommendation for medical school from John Lawrence. Although I did not fully appreciate it at the time, I had the ideal background to succeed in nuclear medicine. After medical school at the University of California, San Francisco, I completed residencies in both internal and nuclear medicine at the University of Washington. The director of nuclear medicine, and my mentor, was Wil Nelp, who also mentored Steve Larson a few years before. My subsequent career included a lot of research, some R01s, and increasing involvement in the Society of Nuclear Medicine. In the 2000s, I was appointed to the Nuclear Medicine Residency Review Committee of the Accreditation Council for Graduate Medical Education (ACGME) and was elected to the ABNM. This put me in a position where I was involved in nuclear medicine education at all levels, from program director to policy maker.

\section{Perspective}

It was apparent by the early 1990s that nuclear medicine was beginning to have difficulty attracting the high-quality resident applicants we had seen in the past. In late 1993, amid increasing discussion about the rapidly rising costs of health care, primary care physicians in some areas of the country tried to reduce costs by ordering fewer imaging examinations. At the University of Washington, volume dropped by $50 \%$ during November 1993. It returned to normal the next month, but a powerful message had been sent and many physicians in nuclear medicine, radiology, and other specialties thought this marked the end of nuclear medicine. At the annual meeting the following June, a single advertisement appeared on the job opportunities board-at King Faisal Hospital (Saudi Arabia).

Also during 1993, the ABNM dropped the requirement from 2 clinical years to 1 year. The apparent impetus was an attempt to increase the number of individuals entering the field. The fact that the number of physicians becoming certified by the ABNM did not increase in the subsequent years suggests that the change was not as effective as had been hoped.

In 2007, the residency requirements changed again, to include 3 years of nuclear medicine after 1 clinical year. Although this change has strengthened the training, it has not sufficiently solved the problem. Over the past 2 decades, nuclear medicine programs, with a few notable exceptions, have been relatively lax in the quality of the applicants accepted. The result has been similar to the economic maxim that bad money drives out the good. The good applicants have avoided nuclear medicine, and we have been left with the less capable. Over these decades, we have failed to train enough high-quality academic physicians to replace the existing 
ones. Increasingly, we are being replaced by radiologists who are perfectly capable of doing an excellent job in clinical nuclear medicine and training but who have little training, tradition, or understanding of how to conduct research.

However, nuclear medicine will not go away. The continuing need for nuclear medicine physicians to interpret studies remains, and numerous new agents are being developed that will need to be ushered through to Food and Drug Administration approval. With few exceptions, successful nuclear medicine physicians will need to have training and board certification in both nuclear medicine and radiology. Pathways are in place to integrate nuclear medicine into the radiology curriculum, by first focusing on radiology and then on 1 year of nuclear medicine, or by first doing nuclear medicine and then 3 years of radiology within a 5-year programthat is, training residents in both specialties in an interleaved fashion. The latter is the optimal approach but is not publicized adequately in most radiology programs. The Janus project described above by Dr. Osborne is an ideal program but is unlikely to be widely available.

The evident solution is to identify the interested, capable, and motivated medical students and educate them about the career pathway of academic nuclear medicine. These individuals could become radiology residents in a combined program and would have the potential to become future leaders in our field. Creating such a program would not be simple. In most medical schools, students are exposed to nuclear medicine in only a 1-hour lecture. The solution would require a dedicated educational effort during medical school training, which requires time and is often politically difficult to arrange. Realistically, the only feasible approach would be to identify interested radiology resident applicants and accept them into a dedicated 5-year program as part of the resident match system. Once radiology residents have decided they are interested in combining nuclear medicine with radiology, it will be essential to have frequent contact and involve them in a long-term research project during their training. Otherwise, they will see conventional radiologists as their role model and will lose interest in nuclear medicine.

Only if radiology program directors are truly invested in transforming the training path can this approach be successful. Efforts at the local level alone are unlikely to bring about this success-real commitment and leadership will be required at the highest levels of the SNMMI, the ABNM, and the ACGME, as well as collaborative efforts with their counterpart radiology organizations.

\section{SAMITT}

\section{Personal Introduction}

Like Dr. Osborne, I received my MD degree from Columbia University College of Physicians and Surgeons. Subsequently, I received my MBA degree from the Wharton School and completed my clinical training in internal medicine at Brigham and Women's Hospital.

I am currently a partner and global provider practice leader at Oliver Wyman after serving as president and chief executive officer of HealthCare Partners, a subsidiary of DaVita HealthCare Partners, and as chief executive officer of Dean Health System, one of the largest integrated delivery systems in the Midwest. I am currently in my fourth year as a commissioner on the Medicare Payment Advisory Commission, and I have a keen interest in the transformation of health care from a volume-based into a valuebased system.

\section{Perspective}

I was invited to contribute to this letter so that the sometimes disparate opinions can be distilled into a set of action items, which I will specify below.

What is remarkable is that the challenge facing the SNMMI and nuclear medicine training programs reflects a parallel trend experienced in many other clinical sectors in the health care industry. When family medicine physicians in my generation pursued residency training, they fully expected that their expertise in adult medicine, pediatrics, hospitalist services, obstetrics and gynecology, and general surgery would consistently be applied to afford a persistent comprehensive clinical portfolio throughout their career. Little did they know that their roles would evolve primarily to adult medicine or pediatrics, with very little reliance on other areas of expertise. Likewise, many U.S. hospitals were constructed with the premise that all of the wide array of cardiovascular procedures, surgical specialties, post-acute care services, long-term intensive care services, and minor-acute/urgent care services would be fully used for years to come, but the use of hospital services for many of these clinical needs has been eroded by the progressive improvement in technologic capabilities, clinical safety protocols, and alternative treatment settings. What all these physicians, disciplines, and societies did not prepare for was the march of clinical advancement, the creation of new technologic modalities, and society's demand for evidence-based solutions that ensure better care at a lower cost. In short, change happens. And such change will inevitably affect physicians and other health care stakeholders, both now and long into the future.

What is also remarkable about the overview Drs. Osborne, Beylergil, and Graham offer above is the general absence of any reference to the patient or the payer or to what the consumers (whether users or funders) expect from the health care community. As our clinical world inevitably also evolves to one that is retailfocused, inclusive of high-deductible and transparent marketplaces for purchasers of health care services and innovative lower-cost clinical disruptors, providers will need to think as retailers do and develop products that meet consumers' needs. As such, what solution would nuclear medicine residency programs and societies develop if charged with starting with the consumer first and determining how best to develop the clinical expertise to deliver high-quality, efficient, evidence-based, low-cost care? In all reality, the solution may mean the erosion (or obsolescence) of some clinical disciplines and the rapid expansion of others. At the end of the day, the goal should be not about preservation of certain clinical disciplines but about what is ultimately in the best interests of the patient.

I agree that there will always be a role (at least in the foreseeable future) for physicians singularly focused on nuclear medicine, but the discipline will likely be a far smaller one characterized by a focus on academics, research, and highly specialized clinical expertise. Those physicians with a love for that discipline should be afforded opportunities to invest their full career in it. Those who wish to have a wider choice of job opportunities will likely see joint training and generalization/diversification as the better avenue to pursue. Again, this trend is not unlike similar choices about optimal paths that other stakeholders in our industry face. Hospitals that see a reduction of inpatient services as the industry shifts to population health must choose between the path of traditionalism as volume player and the path of innovation and transformation as a value leader.

I believe that any action items pursued should have the following general principles: first, the discussion about optimal steps should focus on what is best for patients and society; second, 
the presumption should be that the world will shift to value and those disciplines offering better outcomes, more accurate diagnostics, greater safety, and lower cost; and third, a solution developed jointly, through the breaking down of silos, will always serve stakeholders better than competing sectors that focus on the success of their own individual silos.

\section{Action Items}

The fusion of the ABR and the ABNM as proposed in different ways by Drs. Osborne, Beylergil, and Graham is a significant opportunity to form an entity that clearly has the potential for additional value. The task force recommendations should proceed with targeted amendments.

The new pathway should dominate the value-added components of the imaging specialties. Advocacy on items sensitive to the Food and Drug Administration and the Centers for Medicare and Medicaid Services should be handled by the most knowledgeable of stakeholders.

When possible, programs should merge or close. Elimination of redundancy and of the training pathways that will not produce practitioners should proceed rapidly. Although change is difficult, one cannot jump a 20-foot gorge with two successive 10-foot jumps.

A top-down strategy will not work. Good change will be effected only with input from all stakeholders. Traditional grassroots may not work, but innovation from stakeholders at the beginning of training needs to be brought to the fore.

\section{CONCLUSION}

It is with some degree of trepidation that we submit these recommendations, as the ABR-ABNM task force proposal has not been adopted at the writing of this letter. The proposal will, however, likely exist in some modified form at the time the letter is published. Our perspective is not an endorsement of the dissolution of the ABNM. We believe that if the board proceeds down this road a very transparent neutral zone will need to exist where some of the most important issues can be addressed. It is clear, for example, that a large gap exists between the 4-month nuclear medicine-trained diagnostic radiology diplomate and the draft nuclear medicine-diagnostic radiology diplomate who will have at least 2 years of nuclear medicine training. Important issues have been raised at recent town hall-style events, but issues also ought to be raised at some juncture by the ABNM diplomates who have training in radiology, nuclear medicine, and internal medicine and should inform the board of the needs of ongoing and future diplomates. Indeed, there will be many changes and iterations over the next 12 months alone as to the future of training the practitioners of our art. It is with that notion intact that we summarize our thoughts. Change is inevitable, and training and the practice of medicine in general are at a point where value and quality are of the utmost importance. The determinant of value and quality will also change how we face the uncertainty of a major change in how future physicians are trained in nuclear medicine. As always, optimism and the opportunity that comes from embracing the challenge always win the day.

\section{REFERENCES}

1. New information: ABR/ABNM task force proposal. SNMMI website. http://www. snmmi.org/NewsPublications/NewsDetail.aspx?ItemNumber=14361. Published July 31, 2015. Accessed September 29, 2015.

2. Larson SM. The Janus project: the remaking of nuclear medicine and radiology. J Nucl Med. 2011;52(suppl 2):3S-9S.
3. Guiberteau MJ, Graham MM. ACR-SNM Task Force on Nuclear Medicine Training: report of the task force. J Am Coll Radiol. 2011;8:388-392.

4. Harolds JA, Oates ME, Metter DF, Guiberteau MJ. Are nuclear medicine residents prepared for employment? A survey-informed perspective. J Am Coll Radiol. 2014;11:221-224.

5. Schelbert HR. Nuclear medicine at a crossroads. J Nucl Med. 2011;52(suppl 2): 10S-5S.

6. McDonald JE, Deloney LA, Jambhekar K. Integrated residency training leading to radiology and nuclear medicine board certification: the Arkansas experience. J Ark Med Soc. 2014;111:38-39.

7. Delbeke D, Royal HD, Frey KA, Graham MM, Segall GM. SNMMI/ABNM joint position statement on optimizing training in nuclear medicine in the era of hybrid imaging. J Nucl Med. 2012;53:1490-1494.

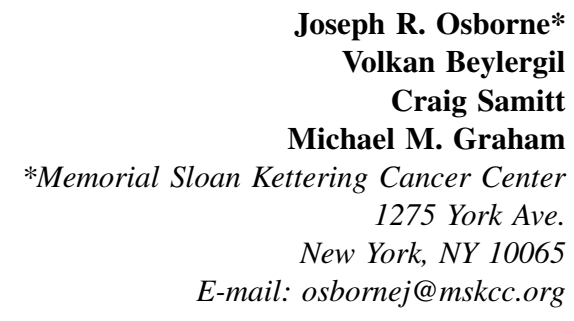

Published online Oct. 1, 2015.

DOI: $10.2967 /$ jnumed.115.165621

REPLY: I thank Dr. Delbeke for allowing me to respond to this interesting letter to the editor from a dual-certified expert who also has a $\mathrm{PhD}$ degree, a trainee, a leader in the field of nuclear medicine, and a health care management consultant. All four perspectives on the future of nuclear medicine (and its independent board) are clearly worth considering.

J.R. Osborne, who is now the director of the Sloan Kettering nuclear medicine training program, concludes that his combined training was the best path to becoming the program director. He points to a gap in training that has widened with the emergence of PET/MRI, a gap that would best be bridged by a joint radiology-nuclear medicine program. He correctly points to one problem that such a construct creates, that is, the loss of board-certified internists, who would no longer be able undergo training in nuclear medicine.

V. Beylergil is a dual-trained nuclear medicine specialist. He trained in radiology because the nuclear medicine job market was dismal and because he wanted to become a better hybrid imager. His qualifications likely make him a very desirable asset for clinical imaging services. A dual training program thus fits his career plans best.

M.M. Graham is a well-known leader in nuclear medicine who held numerous positions in which he attempted to shape the nuclear medicine curriculum. He argues that nuclear medicine is here to stay as evidenced by a plethora of emerging PET imaging probes as well as the advent of effective receptor-targeted radionuclide therapies (theranostics). He correctly points to training pathways that are already in place to integrate nuclear medicine into the radiology curriculum, something that, in his view, is insufficiently publicized. He proposes to identify exceptional talents through the radiology match, nurture them through close interactions while they undergo the required 3 years of radiology training, and then move them into the nuclear medicine program to train the next generation of academic leaders.

These three writers share the belief that a combined training program is desirable and useful to educate predominantly clinically oriented imaging experts. Dr. Graham notes that such a program already exists. 